
\title{
25 Research Soure \\ Lymph Nodes Dissection Guided by Nano-carbon in Laparoscopic Rectal Cancer Surgery
}

\section{Anqi Wang ( $\sim 282674051 @ q q . c o m$ )}

Shanghai Changzheng Hospital

\section{Ce Bian}

Shanghai Changzheng Hospital

\section{Beibei Zhang}

Shanghai Tongren Hospital: Tongren Hospital Shanghai Jiaotong University School of Medicine

Haiyang Zhou

Shanghai Changzheng Hospital

Jian Zhang

Shanghai Changzheng Hospital

\section{Research Article}

Keywords: Nano-carbon,Lymph nodes dissection,Laparoscopic rectal cancer surgery

Posted Date: September 3rd, 2021

DOl: https://doi.org/10.21203/rs.3.rs-864580/v1

License: (c) (1) This work is licensed under a Creative Commons Attribution 4.0 International License. Read Full License 


\section{Abstract}

\section{Background}

To evaluate the role of nano-carbon as a tool for achieving adequate lymph nodes (LNs) dissection and to compare the number of LNs retrieved with and without the use of nano-carbon in laparoscopic rectal cancer surgery.

\section{Methods}

Prospective data collected from September 2018 to August 2019 was retrospectively analyzed. 35 rectal cancer patients received nano-carbon guided laparoscopic rectal cancer surgery were compared with 35 patients without nano-carbon guidance in the evaluation of number of LNs harvest, as well as whether adequate number of LNs retrieved (defined as $\geq 12$ ).

Results

The average number of LNs harvest was significantly higher in the nano-carbon group than that in the control group (16.4 vs. $12.1, \mathrm{P}=0.008$ ). In patients received neoaduvant therapy, the average number of LNs harvest was also significantly higher in the nano-carbon group than that in the control group (13.8 vs. 7.4, $\mathrm{P}=0.011$ ). The percentage of adequate $\mathrm{LNs}$ retrieval was higher in the nano-carbon group than that in the control group ( $82.9 \%$ vs. $62.9 \%, P=0.038)$. In patients received neoaduvant therapy, the percentage of adequate LNs retrieval was much higher in the nano-carbon group than that in the control group $(75.0 \%$ vs. $28.6 \%, P=0.021)$.

\section{Conclusion}

The use of nano-carbon increases the number of LNs harvested compared with the conventional procedure in laparoscopic rectal cancer surgery.

\section{Introduction}

In the current TNM staging system, nodal classification can be determined only when the total number of lymph nodes (LNs) retrieved exceeds 12 for patients with rectal cancer ${ }^{1}$. Insufficient LNs dissection and retrieval have been found to result in poorer survival after surgery ${ }^{2}$. Nano-carbon has been applied recently to facilitate LNs dissection. These particles enter the lymphatic vessels, accumulate in the LNs, and stain them black. We have recently demonstrated the feasibility of using nano-carbon as a tracer to facilitate apical LNs dissection in laparoscopic rectal cancer surgery ${ }^{3}$. The aim of this study is to evaluate the role of nano-carbon as a tool for achieving adequate LNs dissection and to compare the number of LNs retrieved with and without the use of nano-carbon in laparoscopic rectal cancer surgery.

\section{Methods}


A retrospective review of a prospectively collected database was performed. The institutional review board approved the study. Written informed consent was obtained from the patients involved in the study. Between September 2018 to August 2019, 35 rectal cancer patients received nano-carbon guided laparoscopic rectal cancer surgery. Briefly, one day before surgery, the patients received a submucosal injection of $1 \mathrm{ml}$ nano-carbon suspension (LUMMY Pharmaceutical Co., Chongqing, China) beside the tumor under colonoscopy (Fig. 1A). For comparison, 35 patients who underwent laparoscopic rectal cancer surgery without nano-carbon guidance were matched for type of surgery, histopathology, neoadjuvant therapy, age and sex. The number of LNs harvest, as well as whether adequate number of LNs retrieved (defined as $\geq 12$ ) were evaluated. Data were analyzed using 1-tailed Fischer exact test or 1tailed $t$ test with unequal variance as appropriate. $\mathrm{P} \otimes 0.05$ was considered statistically significant.

\section{Results}

Thedraining LNs could be clearly visualized and dissected under laparoscope (Fig. 1B,C). The harvesting process was facilitated as the LNs were stained black because of nano-carbon deposition (Fig. 1D). The clinical and pathological characteristics were comparable between the two groups. The average number of LNs harvest was significantly higher in the nano-carbon group than that in the control group (16.4 vs. $12.1, P=0.008)$. In patients received neoaduvant therapy, the average number of $L N s$ harvest was also significantly higher in the nano-carbon group than that in the control group (13.8 vs. $7.4, P=0.011)$. The percentage of adequate LNs retrieval ( $\geq 12$ lymph nodes harvest) was higher in the nano-carbon group than that in the control group $(82.9 \%$ vs. $62.9 \%, P=0.038)$. In patients received neoaduvant therapy, the percentage of adequate LNs retrieval was much higher in the nano-carbon group than that in the control group $(75.0 \%$ vs. $28.6 \%, P=0.021)$ (Table 1$)$. 
Table 1

Clinical and pathological comparisons between the two groups

\begin{tabular}{|llll|}
\hline Variables & Nano-carbon $(\mathbf{n = 3 5})$ & Control $(\mathbf{n}=\mathbf{3 5})$ & P-value \\
\hline Age, $y$ & $58.6 \pm 13.4$ & $57.2 \pm 15.2$ & 0.684 \\
\hline Gender (Male/ Female) & $23 / 12$ & $25 / 10$ & 0.797 \\
\hline BMI & $22.8 \pm 2.9$ & $23.1 \pm 3.2$ & 0.682 \\
\hline Tumor size, mm & $3.1 \pm 1.4$ & $3.2 \pm 1.7$ & 0.789 \\
\hline Tumor location (Low/Middle/Upper) & $10 / 15 / 10$ & $12 / 14 / 9$ & 0.874 \\
\hline Tumor differentiation (Well/Moderate/Poor) & $5 / 21 / 9$ & $3 / 26 / 6$ & 0.442 \\
\hline Depth of invasion (T0/T1/T2/T3/T4) & $2 / 3 / 11 / 16 / 3$ & $3 / 4 / 7 / 17 / 4$ & 0.843 \\
\hline LNs metastasis (N0/N1/N2) & $23 / 10 / 2$ & $25 / 7 / 3$ & 0.666 \\
\hline Neoaduvant therapy (with/without NAT) & $12 / 23$ & $14 / 21$ & 0.495 \\
\hline Type of Surgery (APR/SPS) & $5 / 30$ & $4 / 31$ & 0.999 \\
\hline Average number of LNs harvest & $16.4 \pm 7.1$ & $12.1 \pm 6.0$ & 0.008 \\
\hline Average number of LNs harvest with NAT & $13.8 \pm 7.2(\mathrm{n}=12)$ & $7.4 \pm 4.5(\mathrm{n}=14)$ & 0.011 \\
\hline$\geq 12$ LNs harvest & $82.9 \%(29 / 35)$ & $62.9 \%(22 / 35)$ & 0.038 \\
\hline$\geq 12$ LNs harvest with NAT & $75.0 \%(9 / 12)$ & $28.6 \%(4 / 14)$ & 0.021 \\
\hline $\begin{array}{l}\text { BMI, body mass index; NAT, neoaduvant therapy; APR, abdominal perineal resection; SPR, sphincter- } \\
\text { preserving surgery; LNs, lymph nodes. }\end{array}$ & & \\
\hline The continuous data are presented as mean \pm standard deviation. & & \\
\hline
\end{tabular}

\section{Discussion}

The use of nano-carbon facilitates the harvesting of LNs from resected specimens, shows a distinct advantage over the conventional methods of LNs retrieval. Altogether, the use of nano-carbon increases the number of LNs harvested compared with the conventional procedure. This is especially relevant in patients received neoaduvant therapy, as previous studies show neoaduvant therapy significantly reduces the number of LNs retrieved, with fewer than 12 detected on average ${ }^{4}$. The retrieval of at least $12 \mathrm{LNs}$ is associated with improved survival in rectal cancer patients received neoaduvant therapy ${ }^{5}$. In present study, by using nano-carbon, the average number of LNs harvest in patients received neoaduvant therapy exceeds 12. Moreover, better visualization of $L N$ s during surgery enables the surgeon to perform safer procedure by preventing injuries when dissecting aroundvessels and nerves, and to provide oncologic benefit through dissectingin the proper plane ${ }^{6}$. 


\section{Conclusions}

Endoscopic peritumoral injection of nano-carbon 1 day before surgery allow for visualization of draining LNs under laparoscope, facilitating lymphadenectomy during laparoscopic rectal cancer surgery.

\section{Abbreviations}

LNs: Lymph nodes

\section{Declarations}

\section{Availability of data and materals}

All data generated or analyzed during this are included in this published article.

\section{Acknowledgements}

Not applicable.

\section{Funding}

This work was supported by the National Natural Science Foundation of China (No. 81571827), Shanghai Rising-Star Program (No. 17QA1405600) and Shanghai Excellent Young Medical Talents Program (No. 2017YQ006).

\section{Contributions}

Zhou $\mathrm{H}$ had full access to all the data in the study and takes responsibility for the integrity of the data and the accuracy of the data analysis. Concept and design: Zhou H and Zhang J. Acquisition, analysis, or interpretation of data: All authors. Drafting of the manuscript: Zhou H. Critical revision of the manuscript for important intellectual content: All authors. Statistical analysis: Zhang B. Obtained funding: Zhou H. Administrative, technical, or material support: Zhou H. Supervision: Zhang J.

\section{Ethics declarations}

\section{Ethics approval and consent to participate}

All the subjects provided written informed consent and we obtained informed consent from all the subjects. 


\section{Consent for publication}

All the participants provided consent for publication

\section{Competing interests}

The authors declare that there are no conflicts of interest

\section{References}

1. Benson AB, Venook AP, Al-Hawary MM, Cederquist L, Chen YJ, CiomborKK,et al. Rectal Cancer, Version 2.2018, NCCN Clinical Practice Guidelines in Oncology. J Natl ComprCancNetw. 2018;16:874-901.

2. Betge J, Harbaum L, Pollheimer MJ, Lindtner RA, Kornprat P, Ebert MP, et al. Lymph node retrieval in colorectal cancer: determining factors and prognostic significance. Int J Colorectal Dis. 2017;32(7):991-8.

3. Xu J, Zhou H, Teng S, Hu Z. Radical apical lymph nodes dissection guided by carbon nanoparticles in laparoscopic rectal cancer surgery - video vignette. Colorectal Dis. 2019 Aug 10.

4. Rullier A, Laurent C, Capdepont M, Vendrely V, Belleannee G, Bioulac-Sage P,et al. Lymph nodes after preoperative chemoradiotherapy for rectal carcinoma: number, status, and impact on survival. Am J Surg Pathol. 2008;32(1):45-50.

5. Wang Y, Zhou M, Yang J, Sun XY. Increased lymph node yield indicates improved survival in locally advanced rectal cancer treated with neoadjuvant chemoradiotherapy. Cancer Med. 2019 Jun 28.

6. Julia K, Emmanouil F, Tim B, Philipp S, Christian W, Arndt H, et al. Association of Plane of Total Mesorectal Excision With Prognosis of Rectal Cancer: Secondary Analysis of the CAO/ARO/AIO-04 Phase 3 Randomized Clinical Trial. JAMA Surg. 2018;153(8):e181607.

\section{Figures}



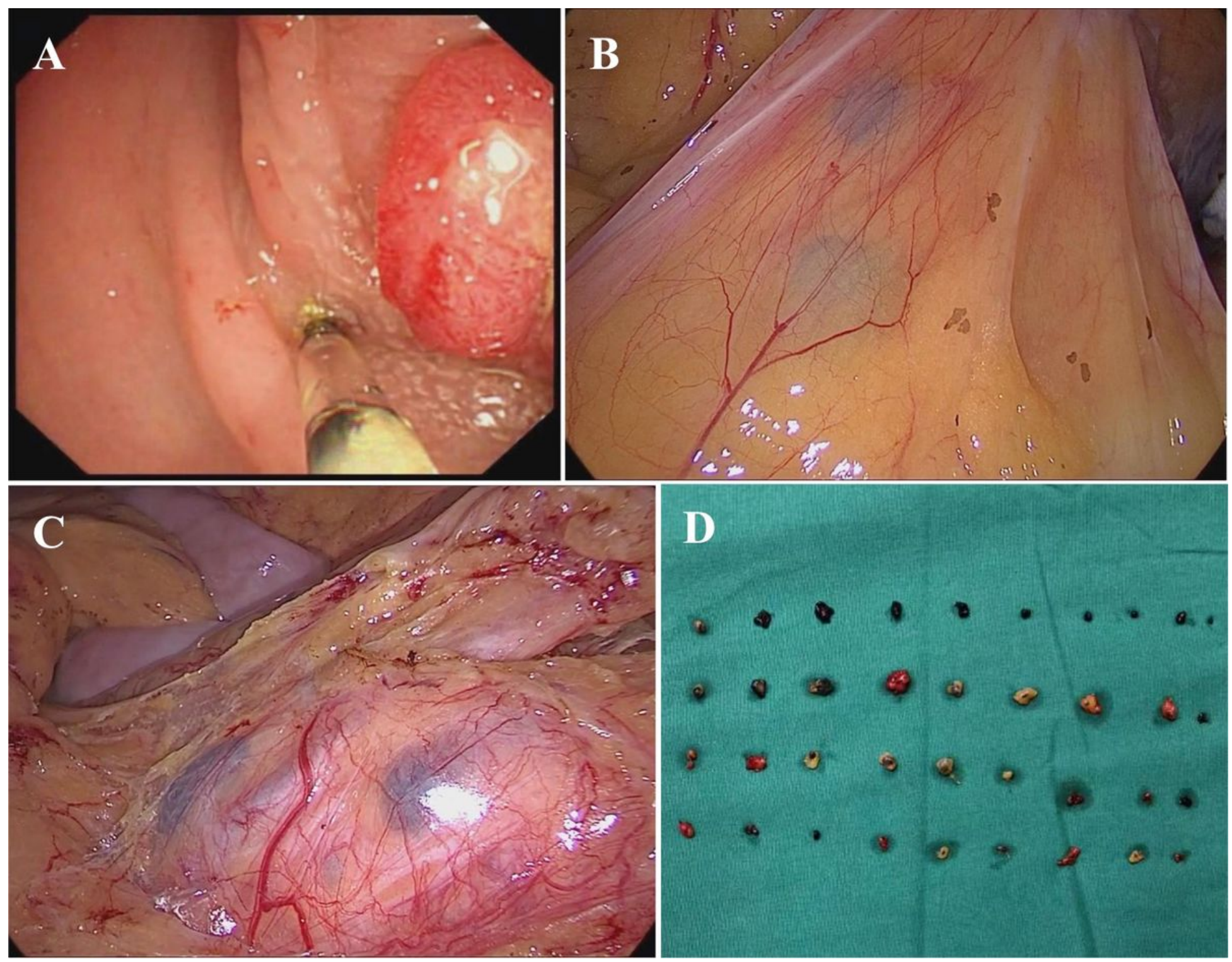

\section{Figure 1}

A, endoscopic peritumoral injection of nano-carbon 1 day before surgery; $B$, nano-carbon black stained mesenteric lymph nodes under laparoscope; C. black stained apical lymph nodes under laparoscope; D. lymph nodes harvested in a patient received nano-carbon guided laparoscopic rectal cancer surgery. 